\title{
Numerical Study of Thermal Conductivity Reduction in Nanolayered Si- Ge Structures by Solving the Equation of Phonon Radiative Transfer
}

\author{
M. Mohamadi ${ }^{*}$, M. A. Mehrabian' ${ }^{1}$ A. Raisi ${ }^{2}$ \\ ${ }^{1}$ Shahid Bahonar University of Kerman, Department of Mechanical Engineering, Faculty of \\ Engineering, Kerman, Iran \\ e-mail: mohamadi.meysam@eng.uk.ac.ir \\ e-mail: mohamadi.meysam@yahoo.com \\ ${ }^{2}$ ShahreKord University, Department of Mechanical Engineering, Faculty of Engineering, \\ ShahreKord, Iran \\ *corresponding author
}

\begin{abstract}
Materials with low thermal conductivity, high electrical conductivity, and high Seebeck coefficient are required for high efficiency solid-state energy conversion. Although semiconductors are the best thermoelectric materials, they rarely have the desired properties. Nanostructures such as superlattices, quantum wires, and quantum dots provide novel methods to improve the solid-state energy conversion efficiency through electron and phonon transport engineering. In this paper a semiconducting superlattice consisting of periodic nano layers of silicon and germanium has been studied. Due to nano scale effects, conductive heat transfer does not satisfy the Fourier's law of thermal conduction, and the equation of phonon radiative transfer has been solved instead. A computational method similar to Discrete Ordinate Method in thermal radiation was implemented, and the equations were solved numerically. The results show that the thermal conductivity of the nano structure is much lower than the macro structures with the same aspect ratio. It was also noticed that with a constant ratio of layers' thicknesses, more reduction in layers' thicknesses causes more temperature jump at the interfaces and consequently more reduction in the effective thermal conductivity, that finally improves the thermoelectric properties. It was also shown that the effective thermal conductivity depends on the density of interfaces per unit length of the superlattice, when the heat flow direction is perpendicular to the layers.
\end{abstract}

Keywords: Solid-State Energy Conversion, superlattices, equation of phonon radiative transfer, nanoscale heat transfer

\section{Introduction}

Nowadays, direct energy conversion is very important for electrical power generation. Implementation of heat sources instead of electrical power for refrigeration is also a serious challenge. Solid state energy conversion by means of thermoelectricity is a direct energy conversion method that can be used for both power generation and refrigeration. Thermoelectric generators operate based on the Seebeck effect, while thermoelectric refrigerators work based 
on the Peltier effect. For example, radioisotope thermoelectric generators (RTGs) are used in space crafts for deep space exploration. These generators directly convert the heat of radio isotope fuel to electricity. The RTGs used in Galileo, Ulysses and Cassini space crafts are so designed that the required heat is provided through plutonium dioxide fuel (238) that totally generates $4400 \mathrm{~W}$ power for RTG. RTG consists of $572 \mathrm{Si}-\mathrm{Ge}$ thermoelectric modules generating $300 \mathrm{~W}$ electrical power. The hot and cold junctions' temperatures are $1000^{\circ} \mathrm{C}$ and $300^{\circ} \mathrm{C}$, respectively. Consequently, it is obvious that the efficiency of these generators is very low (less than 10\%). A method to increase the efficiency is to improve the properties of thermoelectric materials that are effective in energy transport.

Figure of merit is representing the efficiency and energy density of thermoelectric materials and can be defined in a nondimensional form as:

$$
Z T=S^{2} \sigma T / k
$$

where $S$ is the Seebeck coefficient, $k$ is the thermal conductivity and $\sigma$ is the electrical conductivity. Electrons and phonons are the energy carriers, causing conductive heat transfer in solids. Phonon is the quantum of vibrational energy of lattices in solids. Insulating materials have low electrical conductivity, and metals have relatively low Seebeck coefficients. Furthermore, thermal conductivity of metals, mostly controlled by electrons, is proportional to their electrical conductivity. So, metals do not have high figures of merit; however, we can find the highest figures of merit in the semiconductors (Chen et al. 2002). In semiconductors, the phonon thermal conductivity, $k_{p}$, is much higher than the electron thermal conductivity, $k_{e}$, and the phonon thermal conductivity can be reduced significantly, while the electron thermal conductivity reduces slightly. A proven approach to reduce the phonon thermal conductivity is through alloying (Ioffe 1957). The mass difference scattering in an alloy reduces the lattice thermal conductivity significantly without much degradation of the electrical conductivity. The traditional cooling materials are alloys of $\mathrm{Bi}_{2} \mathrm{Te}_{3}$ with $\mathrm{Sb}_{2} \mathrm{Te} e_{3}$ (such as $B i_{0.5} S b_{1.5} T e_{3}$, p-type) and $\mathrm{Bi}_{2} \mathrm{Te}_{3}$ with $\mathrm{Bi}_{2} \mathrm{Se}_{3}$ (such as $\mathrm{Bi}_{2} \mathrm{Te}_{2.7} \mathrm{Se}_{0.3}$, n-type), with a $\mathrm{ZT}$ approximately equal to one at room temperature (Rowe 1995). Materials with $Z T \sim 1$ cannot compete with the conventional fluid-based cooling and power generation technologies. For this purpose, we must produce materials with ZT of 3 or more (Casian et al. 2004 and Alam et al. 2012). Fortunately, based on electron and phonon transport engineering, great improvements for increasing $Z T$ have been achieved. For example, the implementation of semiconductor nano composites with fixed electron transport properties, may result in higher values of $Z T$ by lowering the phonon thermal conductivity (Yang et al. 2005).

So far, the study of heat transfer in microscales, and thermal properties of microstructures has been of interest to researchers and scientists. Casimir verified scale effects on phonon transport at low temperatures (Casimir 1938). Holland investigated the scattering of phonons in semiconductors through thermal conductivity study (Holland 1964). Pohl and Stritzker studied the scattering of phonons on crystal surfaces (Pohl et al. 1982). They investigated the scattering of phonons on crystal surfaces through measuring the thermal conductivity of crystalline insulations at very low temperatures. Tom, et al. studied the surface scattering and radiative heat transfer of phonons (Tom et al. 1988). They used crystals with high quality specular surfaces so that the chance of diffusive scattering was very low. Joshi and Majumdar, 1993 studied the transient ballistic and diffusive phonon heat transfer in thin layers (Joshi et al. 1993). Chen studied the effects of size and interface on thermal conductivity of superlattices and structures with thin alternate layers in parallel direction with layers (Chen 1997). Raisi and Rostami investigated the transient heat transfer in a superlattice composed of alternate layers of GaAs/AlAs in transverse direction (Raisi et al. 2002). Yang and Chen modeled the effective thermal conductivity of a two-dimensional alternate nanocomposite consisting of squared 
nanowires of silicon in a germanium substrate. They solved the equations of Boltzmann transfer and phonon radiative transfer and attained the thermal conductivity of the nanocomposite in the direction of heat transfer, which is perpendicular to the axes of nanowires. Lukes and Tien used molecular dynamics simulation to achieve thermal conductivity (Lukes et al. 2004). They studied a thin nano porous layer and searched for the effects of nano pores location on thermophysical properties, especially the thermal conductivity. Yang, et al. obtained the longitudinal thermal conductivity of a nanocomposite consisting of circular nanowires of silicon in germanium substrate (Yang et al. 2005). Nabovati et al. employed the Lattice Boltzmann Method (LBM) to study the phonons transport in crystalline structures (Nabovati et al. 2011). Garg and Chen computed the thermal conductivity of Si-Ge superlattice from density functional perturbation theory, using relaxation times that include both anharmonic and interface roughness effects (Garg et al. 2013). Tian et al. (2014) applied the atomistic Green's function method to calculate the phonon transmission across Si-Ge superlattices. They have shown that there is an optimum length, resulting from the competition between the coherence of lowfrequency phonons and incoherence of high-frequency phonons caused by interface scattering when anharmonicity is negligible (Tian et al. 2014). Sellitto used a phonon-hydrodynamic approach to obtain a simple mesoscopic model allowing estimating the effective thermal conductivity of a quantum dot superlattice, sketched as a solid matrix of silicon with inclusions of small ellipsoidal obstacles of germanium (Sellitto 2015). Hua and Cao studied the ballistic and diffusive heat conduction in nanostructures. They derived a thermal conductivity model for multiply-constrained nanostructures, from the phonon Boltzmann transport equation and they found that their model agrees well with Monte Carlo simulation and experimental data for silicon nano films and nano wires (Hua et al. 2016).

In this research, the phonon radiative transfer equation will be solved numerically to obtain the temperature distribution and the effective thermal conductivity of Si-Ge superlattices. To the best of authors' knowledge, the effect of the density of interfaces per unit length of superlattices has not been reported in the literature, thus, the main purpose of this research is to investigate the influence of this useful parameter on the effective thermal conductivity of the semiconductor superlattices.

\section{Research Perspective}

In this research, a semiconductor superlattice consisting of periodic thin layers of silicon and germanium as shown in Fig. 1 is studied. The primary goal is to study the steady state heat transfer flow perpendicular to the layers of superlattice, with fixed temperature at both ends. Temperature changes and thermal conductivity diagrams for different values of layers' thicknesses have been drawn covering a wide range of Knudsen numbers. Comparing the thermal conductivity in superlattices with similar structures having macro dimensions and the dependence of thermal conductivity on the interfacial density per unit length are other important goals of this paper.

\subsection{Governing Equations}

The conventional continuum-based physical relations describing heat and fluid flow in bulk systems, such as the Navier-Stokes and Fourier equations, do not apply at microscopic scales (Chen 2005). With the macroscopic dimensions and scales, the thermal conductivity may be computed based on the kinetic theory of gases using the following relation:

$$
k=\left(\frac{1}{3}\right) C v \Lambda
$$




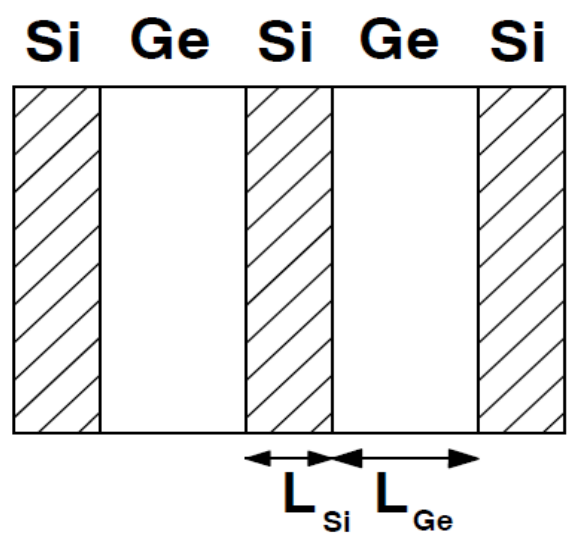

Fig. 1. A semiconductor superlattice consisting of silicon-germanium layers

Where $C$ is the volumetric specific heat, $v$ the phonon group velocity, and $\Lambda$ the phonon mean free path. If the characteristic length is nearly equal or smaller than $\Lambda$, due to the ballistic nature of phonons, this relation is not valid and Fourier's law is not applicable. Fourier's law fails to predict the thermal conductivity of nano structures. More precisely, for a local temperature difference when Fourier's law is not applicable, the thermal conductivity concept is meaningless (Casimir, 1938), but that is still a suitable parameter and can be used to measure the size effects in nano structures. In other words, the thermal conductivity is not an inherent property, but a structural property. Because the characteristic length is sufficiently larger than the coherence length in this research, the wavy nature of phonons can be neglected and they are treated as particles. Therefore, the Boltzmann Transport Equation (BTE) is used as the basic governing equation. Because the characteristic length in this problem is nearly equal or smaller than the phonons mean free path, the ballistic behavior of phonons is dominant. Thus, with implication of phonon radiative intensity idea, we can solve the Equation of Phonon Radiative Transfer (EPRT), which is a differential-integral equation. The BTE with single relaxation time assumption (BGK assumption (Bhatnagar et al., 1954)) and without any external force is expressed as:

$$
\frac{\partial f}{\partial t}+v \cdot \nabla f=\frac{f^{0}-f}{\tau_{r}}
$$

Where $f$ is the phonons' distribution function, $v$ the phonons' group velocity, $\tau_{r}$ the relaxation time, and the superscript 0 shows the equilibrium state. The total phonon intensity defined as follows can be substituted for phonons' distribution function:

$$
I_{i}=\frac{1}{4 \pi} \sum_{m} \int_{0}^{\omega_{\max }}\left|v_{m i}\right| f \hbar \omega D_{m i}(\omega) \mathrm{d} \omega
$$

In this relation the subscript $i$ indicates the material ( $i=1$ refers to germanium and $i=2$ refers to silicon), $D(\omega)$ is the density of states per unit volume, $f$ the phonons' distribution function, $\hbar$ the Plank's constant, $\left|v_{m i}\right|$ the phonons' group velocity magnitude, $\omega$ the phonons' frequency, $\omega_{\max }$ the phonons' maximum frequency for each polarization, and the subscript $m$ shows three polarizations of phonons. In steady conditions, using the total phonon intensity, Eq. (3) can be written as follows: 


$$
\begin{gathered}
v_{x} \frac{\partial I}{\partial x}+v_{y} \frac{\partial I}{\partial y}+v_{z} \frac{\partial I}{\partial z}=\frac{I_{0}-I}{\tau_{r}} \\
v_{x}=v \cos \theta \quad v_{y}=v \sin \theta \cos \phi \quad v_{z}=v \sin \theta \sin \phi
\end{gathered}
$$

After substitution and simple manipulations, the equation of phonon radiative transfer can be written as:

$$
\cos \theta_{i} \frac{\partial I_{i}}{\partial x}+\sin \theta_{i} \cos \phi_{i} \frac{\partial I_{i}}{\partial y}+\sin \theta_{i} \sin \phi_{i} \frac{\partial I_{i}}{\partial z}=\frac{I_{0 i}-I_{i}}{\Lambda_{i}}
$$

Where $\Lambda_{i}$ is the average phonons' mean free path of each material, the product of relaxation time and group velocity magnitudes, and $I_{0 i}$ is the equilibrium phonon intensity defined as:

$$
I_{0 i}(x, y, z)=\frac{1}{4 \pi} \int_{4 \pi} I_{i}(r, \Omega) d \Omega=\frac{1}{4 \pi} \int_{0}^{2 \pi} \int_{0}^{\pi} I_{i}(x, y, z, \theta, \phi) \sin \theta d \theta d \phi
$$

As shown in Fig. 2, $\theta$ and $\phi$ are the polar and azimuthal angles, respectively.

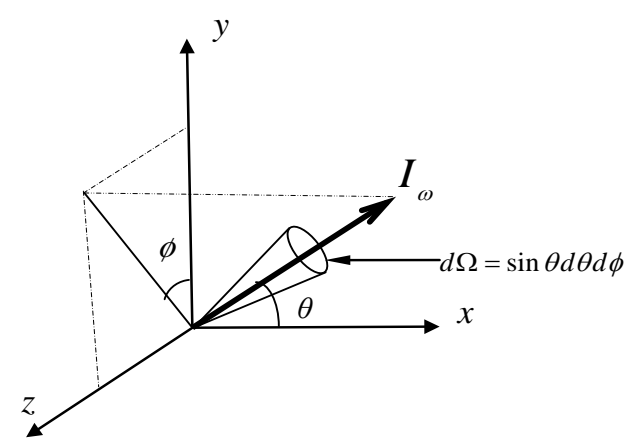

Fig. 2. The coordinate system, the polar, and azimuth angles

\subsection{Domain of solution}

According to Fig. 3, the domain of solution consists of a Si layer between two Ge half layers constructing a periodic unit cell of superlattice. Then $1 \mathrm{~K}$ temperature difference is applied to both sides of the unit cell, while steady conditions prevail. After solving the temperature field and modeling the thermal resistance, we can see how the effective thermal conductivity is reduced. Three types of nodes exist in the solution domain. The first type is the typical nodes present in Si or Ge media, the second type is the nodes on the boundaries, and the third type is the interfacial nodes. For each type of nodes, we should implement the appropriate equation to get $I(x, \theta), I_{0}(x)$, and finally solve the temperature field in the entire domain. 


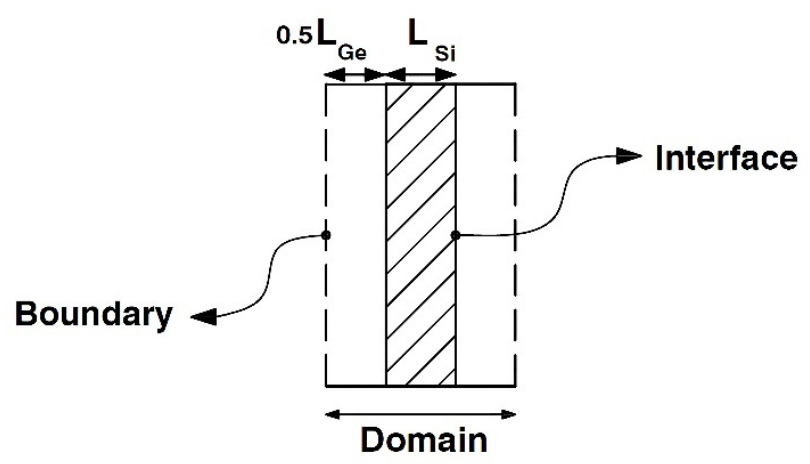

Fig. 3. Solution domain consisting of a Si layer and two Ge half layers

\subsection{Typical nodes}

For these nodes, Eqs. (6) and (7) are simplified; in one dimensional case the following equations are obtained:

$$
\begin{gathered}
\cos \theta_{i} \frac{\partial I_{i}}{\partial x}=\frac{I_{0 i}-I_{i}}{\Lambda_{i}} \\
I_{0 i}(x)=\frac{1}{4 \pi} \int_{4 \pi} I_{i}(r, \Omega) d \Omega=\frac{1}{2} \int_{0}^{\pi} I_{i}(x, \theta) \sin \theta d \theta
\end{gathered}
$$

The numerical method used to solve this equation is similar to discrete ordinate method in radiation heat transfer. Due to the positive or negative directions of phonon transfer as shown in Fig. 4, the differential term in Eq. (8) should be forward or backward differenced, respectively:

$$
\cos \theta_{i} \frac{\partial I_{i}^{+}}{\partial x}=\cos \theta_{i} \frac{I_{i}^{+}-I_{i-1}^{+}}{\Delta x} \quad \cos \theta_{i} \frac{\partial I_{i}^{-}}{\partial x}=\cos \theta_{i} \frac{I_{i}^{-}-I_{i+1}^{-}}{\Delta x}
$$

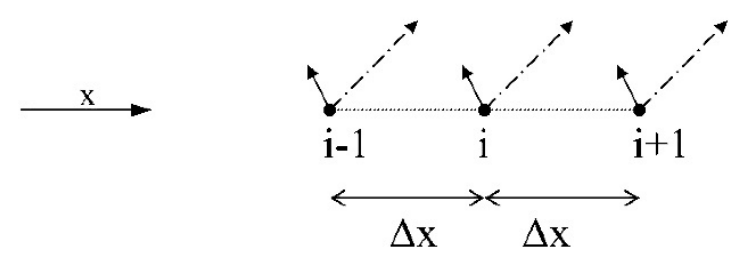

Fig. 4. Forward and backward differencing based on phonon directions

\subsection{Boundary nodes}

From a mathematical point of view, the structure of the Boltzmann transport equation is such that we need to know the boundary conditions only on the boundaries that phonon intensities enter the domain. Therefore, in Fig. 3 intensities in positive $x$ direction should be known for the left boundary, and for the right boundary we should know the intensities in negative $x$ direction. 
Therefore, at boundaries $x=0$ and $x=L_{G e}+L_{S i}$ we use periodic boundary condition based on energy conservation in $x$ direction. In equilibrium state, phonon intensities are isotropic and independent of direction. However, if there is a heat flow that is an indication of thermal nonequilibrium condition, phonon intensities will be distorted.

From a physical point of view, the periodic boundary condition (Yang et al. 2004) in the $x$ direction means that the distortion of phonon intensity in any direction in the boundary $x=0$ is the same as that in the boundary $x=L_{G e}+L_{S i}$. Therefore, this condition will be defined as follows:

$$
I(0, \theta)-I_{0}(0)=I\left(L_{G e}+L_{S i}, \theta\right)-I_{0}\left(L_{G e}+L_{S i}\right)
$$

This condition conserves the total heat flux in $\mathrm{x}$ direction.

\subsection{Interface nodes}

We assume that the phonon scattering at the interfaces is diffusive, thus the transmission and reflection coefficients will be defined using the diffuse mismatch model, DMM.

$$
R_{12}+T_{12}=1 \quad T_{21}=R_{12} \quad T_{12}=\frac{C_{2} v_{2}}{C_{1} v_{1}+C_{2} v_{2}}
$$

The DMM is based on the fact that the phonon scattering is totally diffusive at interfaces. This model assumes that phonons arrive at interfaces from different directions. Therefore, virtually we can assume that a phonon stays at interface and the direction of its motion towards region 1 or 2 is not predictable. Distribution of phonon intensity is shown in Fig. 5 qualitatively.

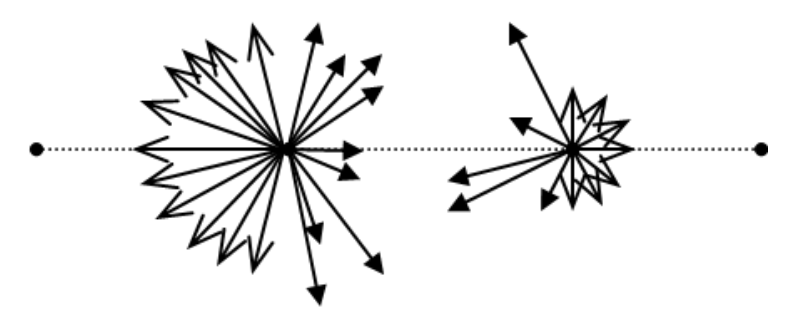

Fig. 5. Diffuse phonon intensity on the interface points

The total diffusive phonon intensity for the first interface of the solution domain is as follows:

$$
\begin{gathered}
I\left(L_{G e} / 2, \theta\right)=-\frac{R_{21}}{\pi} \int_{0}^{2 \pi} \int_{\frac{\pi}{2}}^{\pi} I_{2}\left(L_{G e} / 2, \theta\right) \cos \theta \sin \theta d \theta d \phi+ \\
\frac{T_{12}}{\pi} \int_{0}^{2 \pi} \int_{0}^{\frac{\pi}{2}} I_{1}\left(L_{G e} / 2, \theta\right) \cos \theta \sin \theta d \theta d \phi
\end{gathered}
$$




\subsection{Temperature field and thermal conductivity modeling}

After achieving the total phonon intensity in the whole domain, we should undergo a process to find the temperature field and to model the effective thermal conductivity in the heat transfer direction.

In nanoscale studies temperature is not a criterion for local thermal equilibrium. However, having $I_{0}(x)$ at each plane of superlattice, temperature could be defined as the local energy density at that plane (Yang et al., 2005) according to the following equation:

$$
T(x)=\frac{4 \pi I_{0}(x)}{C|v|}
$$

Then, by integrating the phonon intensity on the entire solid angle we can find the rate of heat transfer in transverse direction as follows:

$$
Q_{x}=\int_{0}^{2 \pi} \int_{0}^{\pi} I(x, \theta) \cos \theta(\sin \theta d \theta d \phi)
$$

Finally, the following equation for modelling the effective thermal conductivity is obtained:

$$
k=Q_{x}\left(L_{G e}+L_{S i}\right) /\left(T(0)-T\left(L_{G e}+L_{S i}\right)\right)
$$

\subsection{Thermal conductivity in macrostructures}

To compute the thermal conductivity in macrostructures of Si-Ge, we can use the Fourier's law of conduction and its results for thermal resistances. For this purpose, by using the conventional heat transfer equations and combined layers resistance, in the absence of contact resistance between the layers, we finally obtain the equivalent thermal conductivity as follows:

$$
\begin{array}{cc}
q=\frac{\Delta T}{R} & R=\frac{L_{1}}{k_{1}}+\frac{L_{2}}{k_{2}} \\
q=k_{\text {eq }} \frac{\Delta T}{\left(L_{1}+L_{2}\right)} & k_{e q}=\frac{\left(L_{1}+L_{2}\right) k_{1} k_{2}}{L_{1} k_{2}+L_{2} k_{1}}
\end{array}
$$

The numerical solution of the governing equations requires to define a two-dimensional matrix for $I(x, \theta)$ and a one-dimensional matrix for $I_{0}(x)$. Then we should discretize the governing equations. For example, using forward and backward differencing, Eq. (8) is converted to:

$$
\begin{gathered}
\left(1+\frac{\Lambda_{i}}{\Delta x} m u(m)\right) I\left(n_{x}, m\right)-\frac{\Lambda_{i}}{\Delta x} m u(m) I\left(n_{x}-1, m\right)=I_{0}\left(n_{x}\right) \\
\left(1-\frac{\Lambda_{i}}{\Delta x} m u(m)\right) I\left(n_{x}, m\right)+\frac{\Lambda_{i}}{\Delta x} m u(m) I\left(n_{x}+1, m\right)=I_{0}\left(n_{x}\right) \\
I_{0}\left(n_{x}\right)=\left(\frac{1}{n_{4}}\right) \sum_{m=1}^{n_{4}} I\left(n_{x}, m\right)
\end{gathered}
$$

The numerical modeling of the equations has been performed using FORTRAN language and iterative method. The associated properties are listed in Table (1). Using the properties 
listed in Table 1, the phonon mean free path for Si and Ge is obtained 268nm and 198nm, respectively.

\begin{tabular}{|l|r|r|r|r|r|r|}
\cline { 2 - 7 } \multicolumn{1}{c|}{} & \multicolumn{1}{c|}{$C\left(\mathrm{~J} / \mathrm{m}^{3} \mathrm{k}\right)$} & $v(\mathrm{~m} / \mathrm{sec})$ & \multicolumn{1}{|c|}{$k(W / \mathrm{mK})$} & $M(\mathrm{~kg} / \mathrm{kmol})$ & $a(\mathrm{~nm})$ & $\rho(\mathrm{kg}$ \\
\hline $\mathrm{Si}$ & 930000 & 1804 & 150 & 28.085 & 0.5431 & 2330 \\
\hline $\mathrm{Ge}$ & 870000 & 1042 & 60 & 72.610 & 0.5658 & 5323 \\
\hline
\end{tabular}

Table 1. Ge and Si properties at room temperature

\section{Results and Discussion}

In this research we investigate the influence of the thickness of Si and Ge thicknesses and their ratio on the temperature distribution across the layers, and on the thermal conductivity. The ratio of the Ge layer thickness to the Si layer thickness is specified in Table 2.

\begin{tabular}{|c|r|r|r|r|r|}
\hline$L_{G e} / L_{S i}$ & 0.283 & 0.754 & 1.131 & 1.697 & 4.524 \\
\hline Ge atomic percentage & $20 \%$ & $40 \%$ & $50 \%$ & $60 \%$ & $80 \%$ \\
\hline
\end{tabular}

Table 2. The ratio of Ge to Si thickness versus Ge atomic percentage

\subsection{The effect of layers' thicknesses on temperature across the layers}

In Figs. 6-10, the temperature profiles across a periodic unit are shown for a total temperature drop of $1 \mathrm{~K}$. In each figure, three different values are chosen for $\mathrm{Si}$ layer thickness $\left(L_{S i}=5,150,16000 \mathrm{~nm}\right)$.

The thickness of Ge layer in each case is related to its atomic percentage. When the Si thickness is $16000 \mathrm{~nm}$ ( $L_{S i}=16000 \mathrm{~nm}$ ), the thickness of layers is much larger than the mean free paths of layers, and therefore the totally diffusive effect is dominant in the conductive heat transfer and the Fourier's law is valid. Thus, in Figs. 11-15 there is not any temperature jump at the interfaces.

When the Si thickness is $150 \mathrm{~nm}\left(L_{S i}=150 \mathrm{~nm}\right)$, then the minimum and maximum Ge thicknesses are $L_{G e, \min }=42.45 \mathrm{~nm}$ and $L_{G e, \max }=678.6 \mathrm{~nm}$, respectively. In this case, both the ballistic and diffusive mechanisms are effective.

Therefore, we can see the temperature gradient across the layers, and the temperature jump at the interfaces, simultaneously. According to Figs. (6) to (10), as the Ge thickness increases from 42.45 to $678.6 \mathrm{~nm}$, the role of ballistic mechanism reduces and the temperature jumps are smaller.

Finally, in the case of $L_{S i}=5 \mathrm{~nm}$, the thickness of both layers is much smaller than the mean free paths, and the mechanism of conductive heat transfer is only ballistic. In fact, in this case the phonons do not have any collision across the layer and they hit the boundaries instead, that causes temperature jumps at the interfaces. 


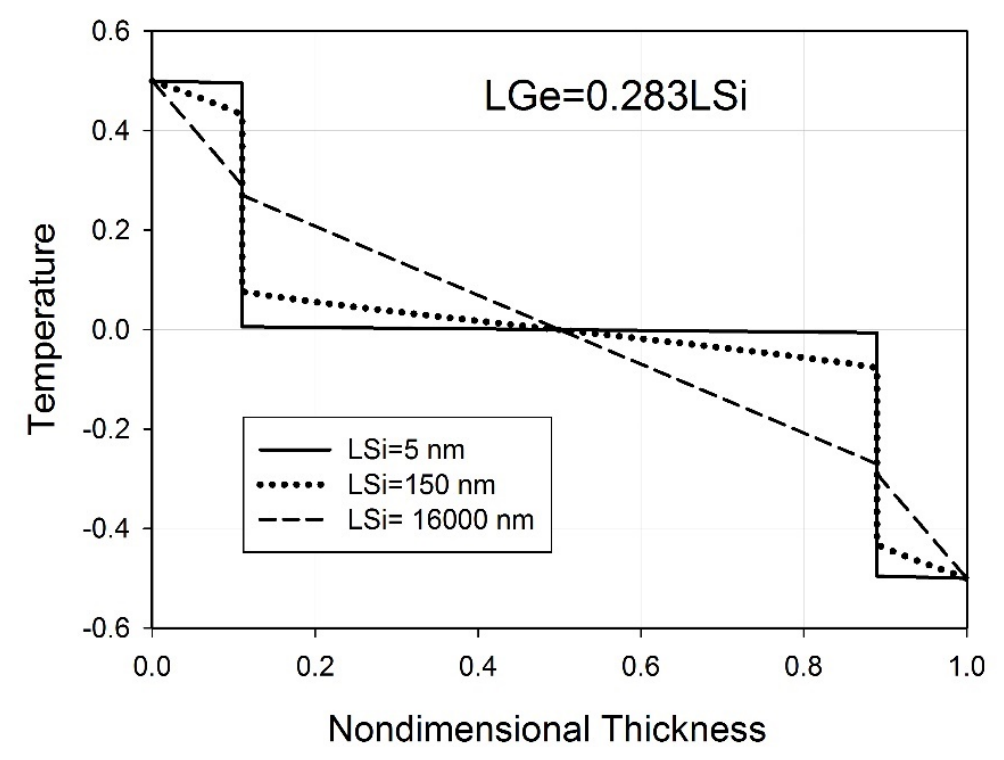

Fig. 6. Temperature profile across the superlattice (20\% Ge)

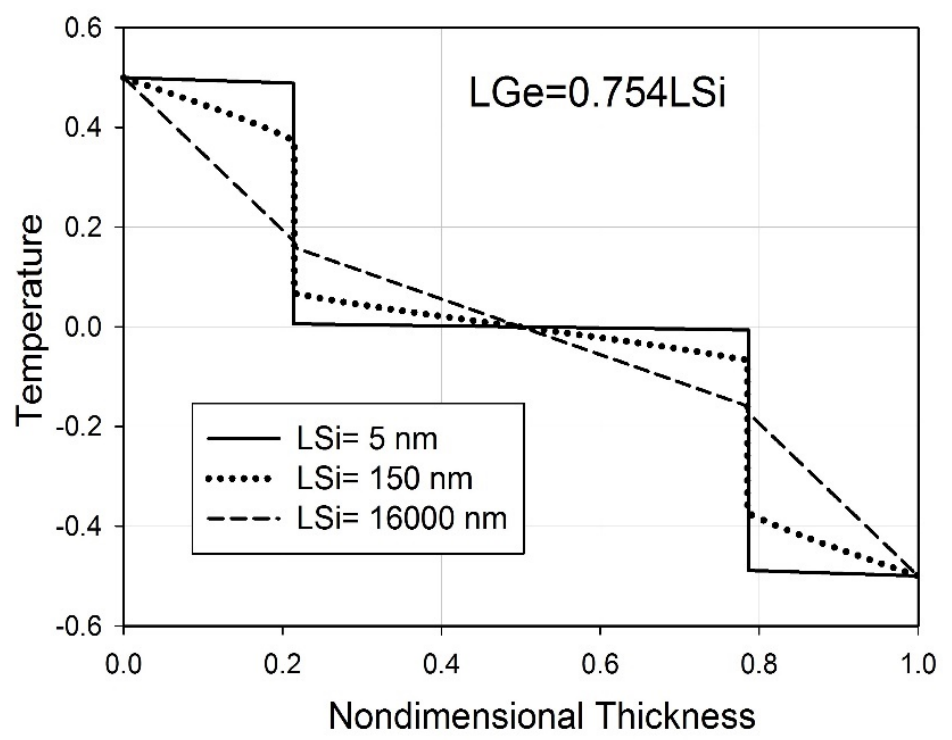

Fig. 7. Temperature profile across the superlattice (40\% Ge) 


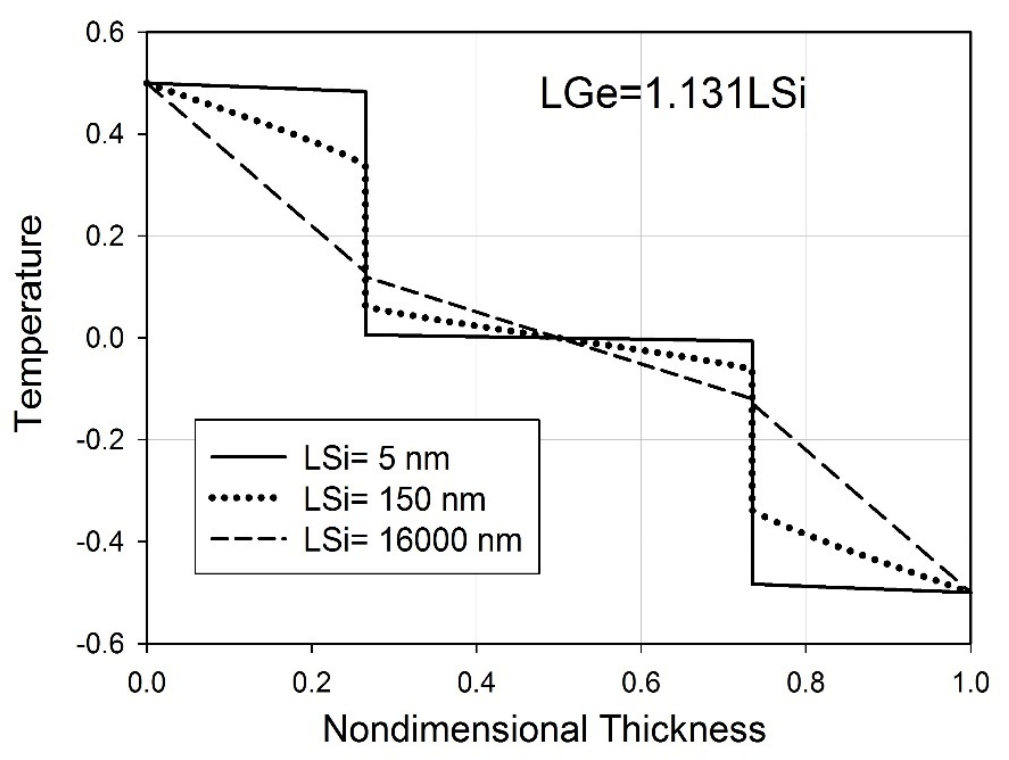

Fig. 8. Temperature profile across the superlattice (50\% Ge)

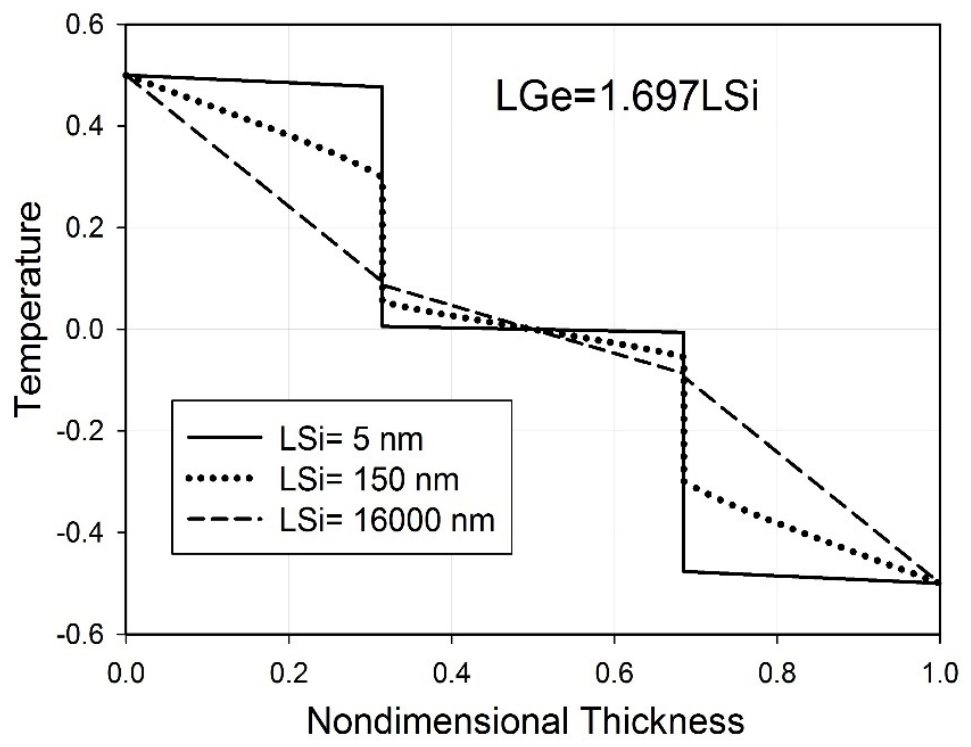

Fig. 9. Temperature profile across the superlattice (60\% Ge) 


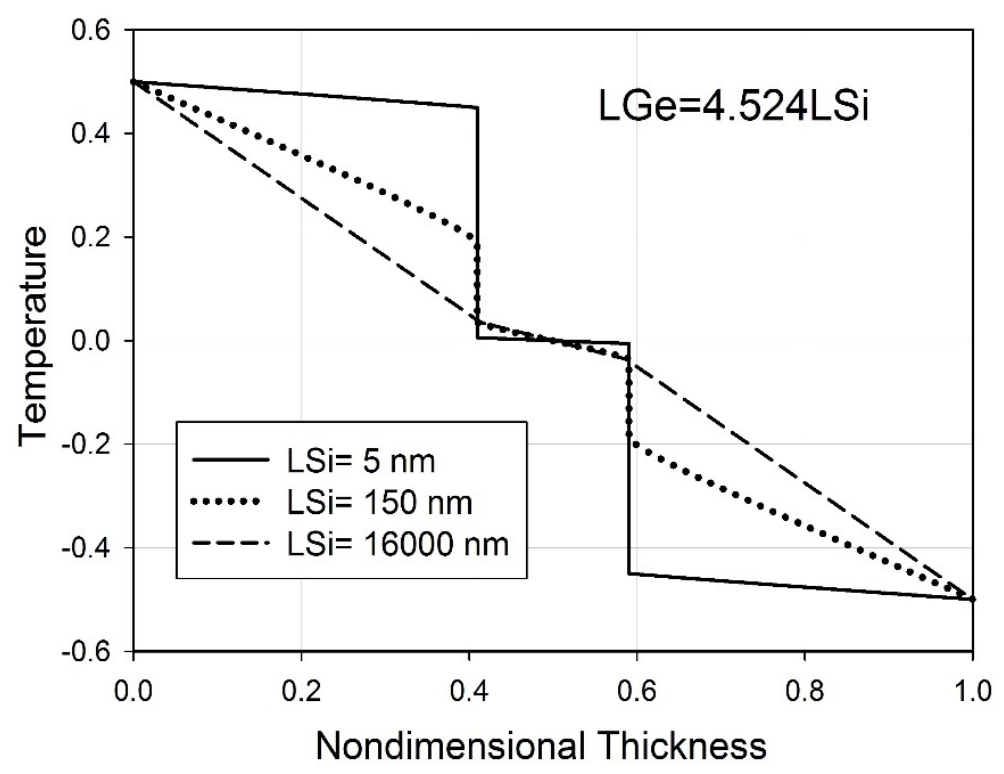

Fig. 10. Temperature profile across the superlattice ( $80 \% \mathrm{Ge})$

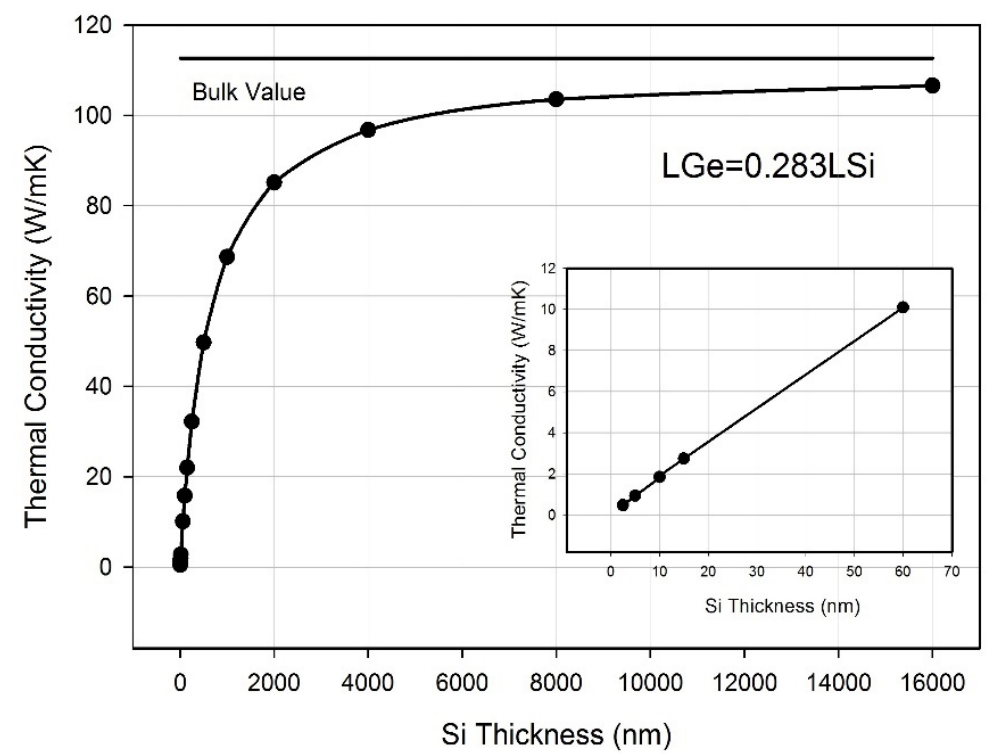

Fig. 11. The effect of Si thickness on thermal conductivity of superlattice (20\% Ge) 


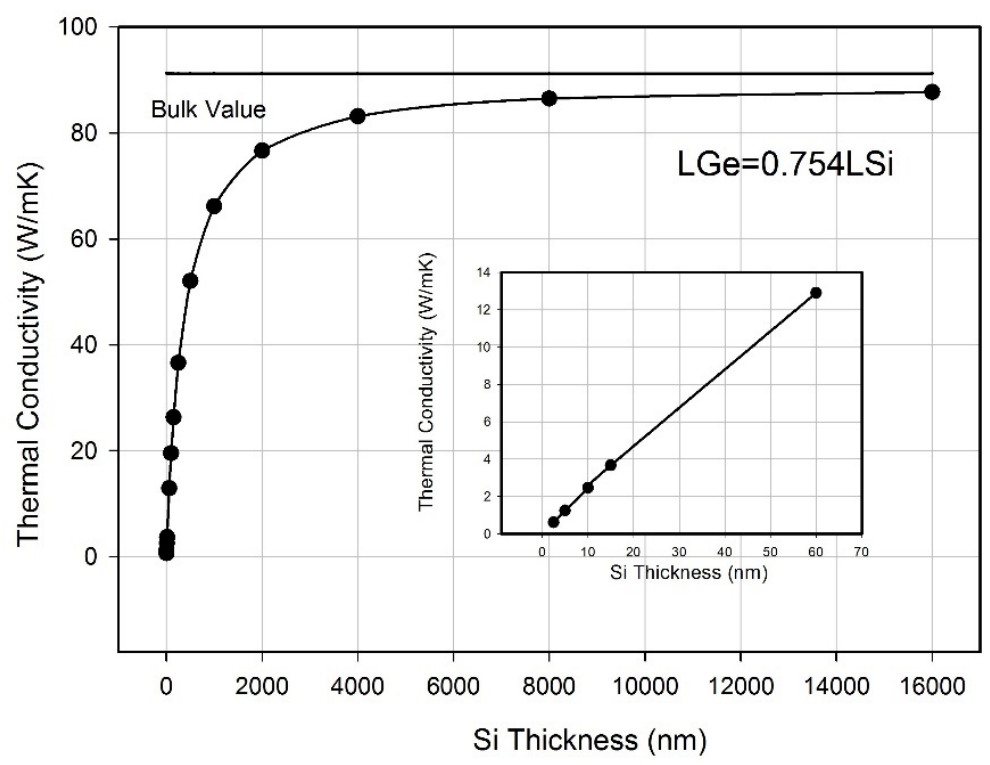

Fig. 12. The effect of Si thickness on thermal conductivity of superlattice (40\% Ge)

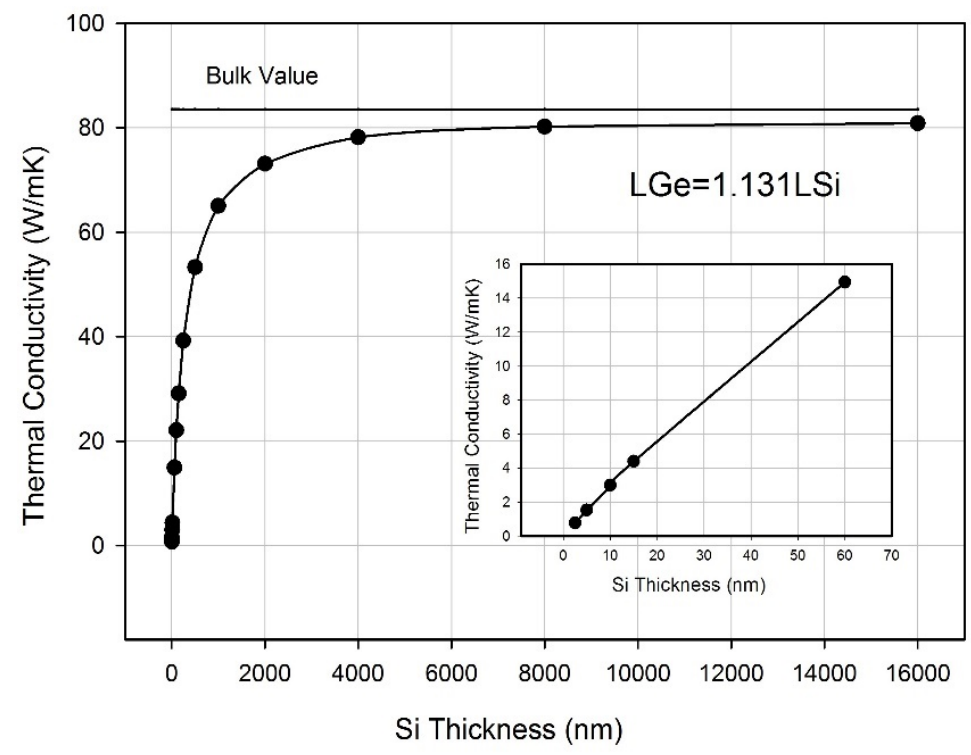

Fig. 13. The effect of Si thickness on thermal conductivity of superlattice (50\% Ge) 


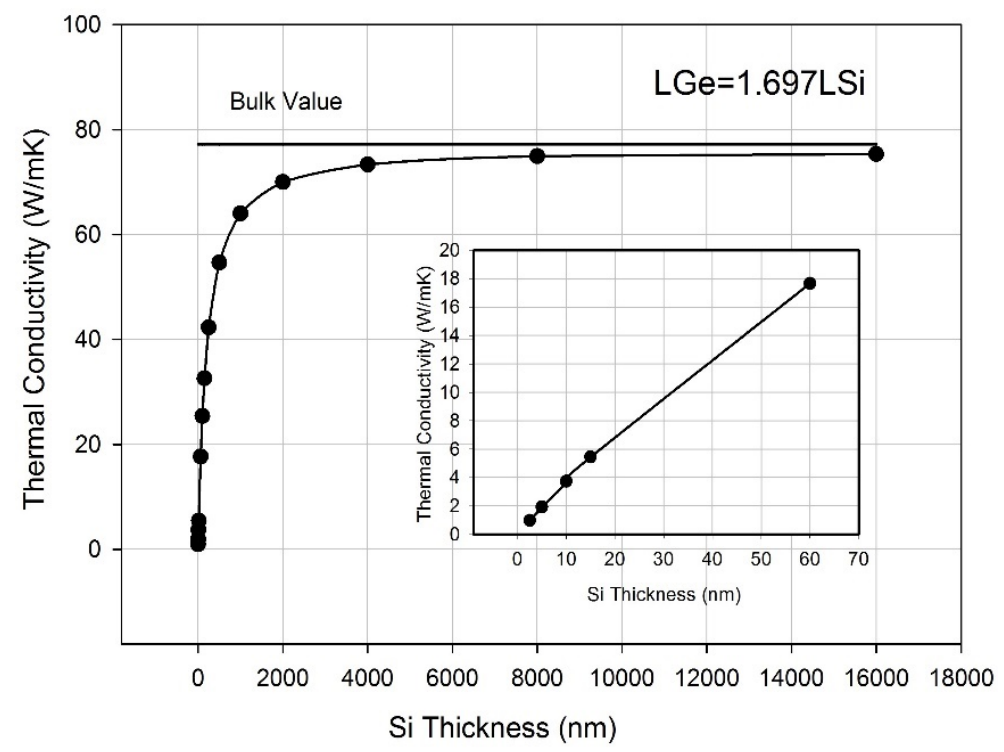

Fig. 14. The effect of Si thickness on thermal conductivity of superlattice (60\% Ge)

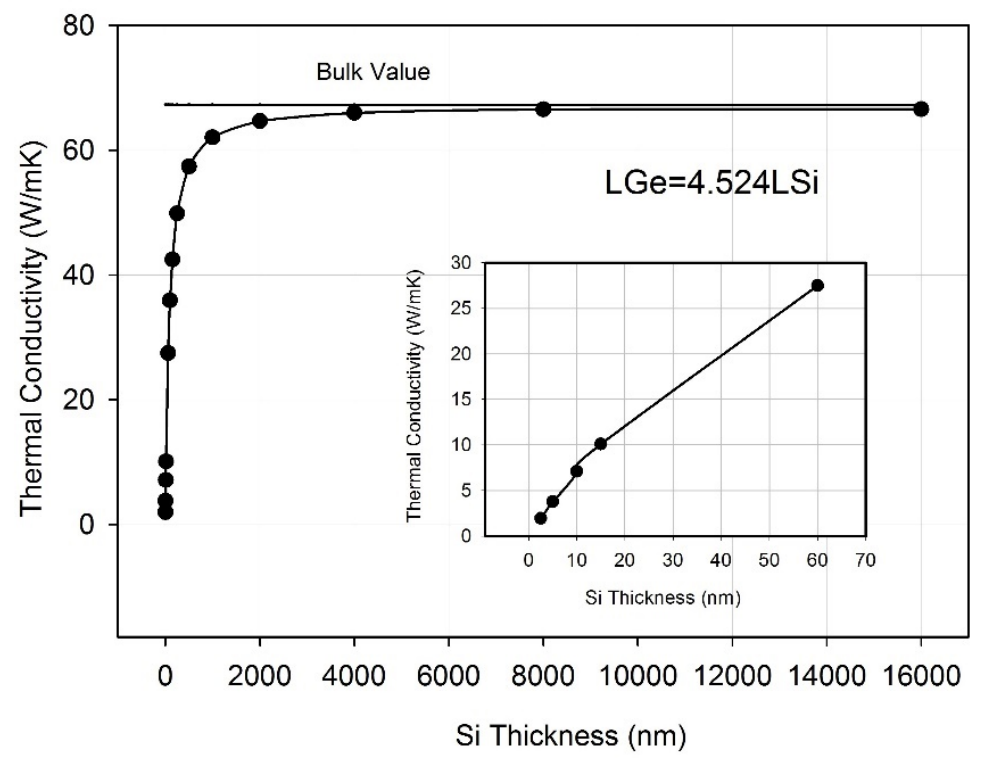

Fig. 15. The effect of Si thickness on thermal conductivity of superlattice ( $80 \% \mathrm{Ge})$

\subsection{The effect of layers thickness on heat flux and thermal conductivity}

Figures 11-15 show the variation of the effective thermal conductivity versus Ge atomic percentage. Each figure represents a specific ratio of the layers' thicknesses. The figures indicate that thermal conductivity increases with increasing the layers' thicknesses. According to these figures, it is obvious that the thermal conductivity depends on layers' thicknesses and as layers thickness reduces, the thermal conductivity also reduces. In fact, when the layers 
thickness is reduced, the effect of thermal boundary resistance and consequently the total thermal resistance is increased, and therefore the heat flux reduces. Finally, according to Eq. (15), the reduction in heat flux causes the reduction in the effective thermal conductivity.

However, with increasing the layers' thicknesses in each figure, the thermal conductivity approaches the macrostructure thermal conductivity value that is computed from Eq. (16) which is an important check point for the code validation. For example, in Fig. 13 that is for $50 \%$ of Ge atomic percentage, the thermal conductivity begins from $0.77 \mathrm{~W} / \mathrm{mK}$ for $2.5 \mathrm{~nm}$ Si layer thickness and finally approaches to $83.5 \mathrm{~W} / \mathrm{mK}$ that is the thermal conductivity of macrostructure.

It is obvious from the figures that by fixing the thickness of Si layer, and simultaneously increasing the thickness of Ge layer, the thermal conductivity will increase. It is also seen that by fixing the thickness of Ge layer and increasing the thickness of Si layer, the thermal conductivity will increase. As mentioned before, when the Si thickness is fixed, the effective thermal conductivity will increase as Ge thickness is increased. For example, in Fig. 16, the Si thickness is $100 \mathrm{~nm}$ and it can be seen that thermal conductivity increases as the Ge thickness grows. For considerable increase in Ge thickness, the thermal conductivity approaches the bulk value of Ge thermal conductivity, namely $60 \mathrm{~W} / \mathrm{mK}$.

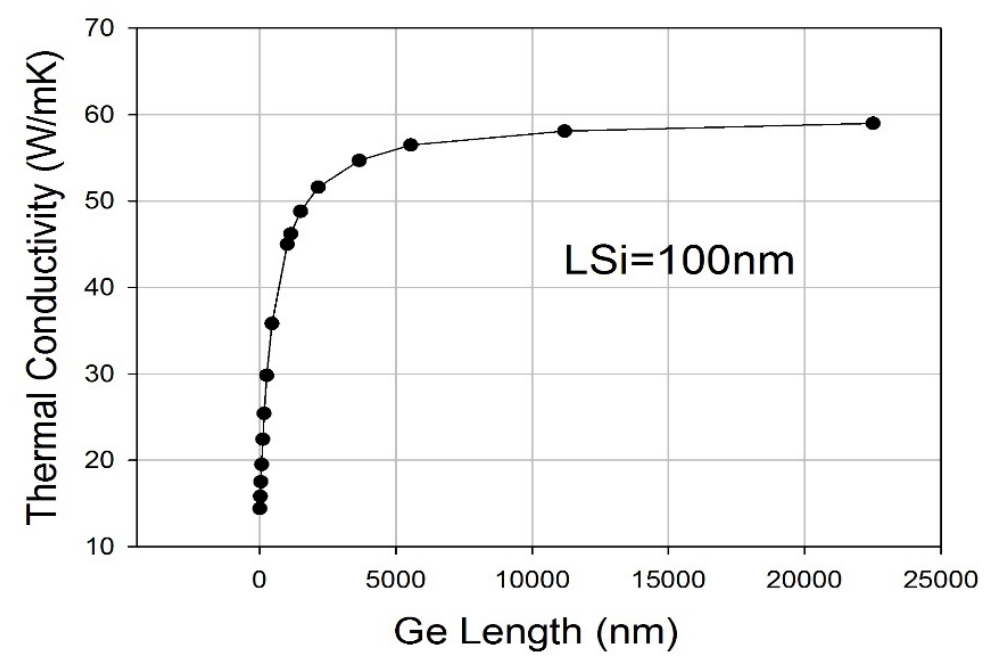

Fig. 16. The variation of thermal conductivity versus Ge length at constant Si thickness

\subsection{The importance of interface density per unit length}

In Fig. 17, the change of thermal conductivity versus a useful parameter named interface density per unit length is demonstrated. It is seen that as the interface density increases, the thermal conductivity reduces.

As shown in Fig. 17, points with different atomic percentages are scattered irregularly between each other. It means that the density of interface per unit length is an important parameter in the study of thermal conductivity in superlattices, when heat flow direction is perpendicular to the layers. We can, therefore, find the thermal conductivity of a Si-Ge superlattice from Fig. 17, when computing the density of the interfaces per unit length. 


\section{Conclusions}

By reducing the thickness of both layers in nanometer scale, it is noticed that the temperature jumps at interfaces grows, while the heat flux and the effective thermal conductivity are reduced. Although, if one of the layers has a nanoscale thickness but the other one has a macroscale thickness, there is no nanoscale effect and the effective thermal conductivity approaches the bulk value of the thicker layer.

The main effective parameter is the interface density per unit length that is more practical than the atomic percentage of the constituents. Without any attention to the ratio of layers, by increasing the interface density, the effective thermal conductivity reduces.

Finally, the reduction in the effective thermal conductivity will increase the figure of merit, and eventually improves the efficiency of thermoelectric materials.

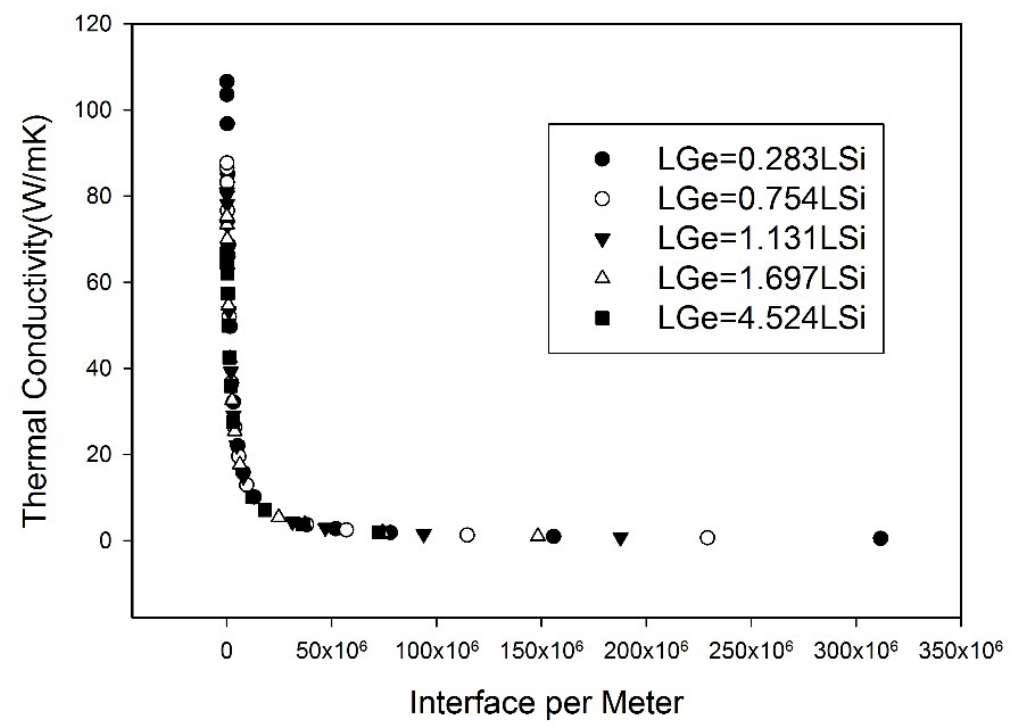

Fig. 17. The variation of thermal conductivity versus interface density per unit length

\section{References}

Alam H, Ramakrishna S (2012). A review on the enhancement of figure of merit from bulk to nano-thermoelectric materials,

Bhatnagar PL, Gross EP, Krook M (1954). A model for collision processes in gases. I. small amplitude processes in charged and neutral one-component systems, Physical Review, 94 (3), 511-525.

Casian A, Dashevsky Z, Kantser V, Scherrer H, Sur I (2004). Thermoelectric figure of merit of n-type PbTe/Pb1-xEuxTe quantum wells, Moldavian Journal of the Physical Sciences, N1.

Casimir HBG (1938). Note on the conduction of heat in crystals, Physica, 5, 495-500.

Chen G (1997). Size and interface effects on thermal conductivity of superlattices and periodic thin films, Journal of Heat Transfer, 119, 220-228. 
Chen G, Shakouri A (2002). Heat transfer in nanostructures for solid-state energy conversion, ASME, 124, 242-252.

Chen G (2005). Nanoscale energy transport and conversion: A parallel treatment of electrons, molecules, phonons and photons, Oxford University Press, New York.

Garg J, Chen G (2013). Minimum thermal conductivity in superlattices: A first-principles Formalism, Physical Review B, 87, 140302(R).

Goldsmid HJ (1964). Thermoelectric refrigeration, Plenum Press, New York.

Holland MG (1964). Phonon scattering in semiconductors from thermal conductivity study, Physical Review, 134, 471-480.

Hua YC, Cao BY (2016). Ballistic-diffusive heat conduction in multiply- constrained nanostructures, International Journal of Thermal Sciences, 101, 126-132.

Ioffe AF (1957). Semiconductor thermoelements and thermoelectric cooling, Infosearch Limited, London.

Joshi AA, Majumdar A (1993). Transient ballistic and diffusive phonon heat transfer in thin film, Journal of Applied Physics, 74, 31-39.

Lukes JR, Tien CL (2004). Molecular dynamics simulation of thermal conduction in nanoporous thin films, Microscale Thermophysical Engineering, 8, 341-359.

Nabovati A, Sellan DP, Amon CH (2011). On the Lattice Boltzmann method for phonon transport, Journal of Computational Physics, 230, 5864-5876.

Pohl RO, Stritzker B (1982). Phonon scattering at crystal surfaces, Physical Review B, 25, No. 6, 3608-3614.

Raisi A, Rostami AA (2002). Unsteady heat transport in direction perpendicular to a doublelayer thin-film structure, Numerical Heat Transfer, Part A, 41, 373-390.

Rowe DM (1995). Handbook of thermoelectrics, CRC Press, Boca Raton.

Sellitto A (2015). A phonon-hydrodynamic approach to thermal conductivity of Si-Ge quantum dot superlattices, Applied Mathematical Modelling, http://dx.doi.org/10.1016/j.apm.2015.03.060.

Succi S (2001). The lattice Boltzmann equation for fluid mechanics and beyond, Oxford University Press, New York.

Tian Z, Esfarjani K, Chen G (2014). Green's function studies of phonon transport across Si/Ge superlattices, Physical Review B, 89, 235307.

Tom K, et al. (1988). Phonon radiative heat transfer and surface scattering, Physical Review B, 38, 7576-7594.

Yang RG, Chen G (2004). Thermal conductivity modeling of periodic two-dimensional nanocomposites, Physical Review B, 69, 195316.

Yang RG, Chen G, Dresselhaus, MS (2005). Thermal conductivity of simple and tubular nanowire composites in the longitudinal direction, Physical Review B. 72, 125418. 